\title{
The influences of polydopamine immersion time on characteristics and performance of polyvinylidene fluoride ultrafiltration membrane
}

\author{
Syawaliah Syawaliah ${ }^{1}$, Nasrul Arahman ${ }^{2}$, Medyan Riza $^{2}$, and Sri Mulyati2,* \\ 1Syiah Kuala University, Doctoral School of Engineering Science, Banda Aceh, Indonesia, 23111 \\ 2Syiah Kuala University, Chemical Engineering Department, Banda Aceh, Indonesia, 23111
}

\begin{abstract}
The Polyvinylidene Fluoride (PVDF) membrane has been prepared by phase inversion method using N,N-dimethylacetamide (DMAc) as solvent and Poly Ethylene Glycol (PEG) as additive. The fabricated membrane was modified by Polydopamine (PDA) coating in concentration of $0.5 \mathrm{mg} / \mathrm{ml}$ and immersion times of 2 hours, 6 hours, and 24 hours. The characteristics and performance of the PVDF membranes before and after the modification are studied in this paper. The result of the water flux experiment showed that the PDA-coated PVDF membranes showcased a higher flux than that of pure PVDF membrane. Scanning Electron Microscopy (SEM) analysis confirmed that the membrane had an asymmetric structure consisting of two layers. There was no significant influence on the addition of PDA to the morphology of the pore matrix because the modification was done by surface coating. Fourier Transform Infrared Spectroscopy (FTIR) analysis showed that PDA was successfully introduced on the surface of PVDF membrane with the appearance of $\mathrm{O}-\mathrm{H}$ from cathecol and $\mathrm{N}-\mathrm{H}$ peaks at wavenumber range of 3300-3600 $\mathrm{cm}^{-1}$. Modification with PDA increased the mechanical strength of the membrane which affirmed by the results of the tensile and elongation at break evaluation.
\end{abstract}

\section{Introduction}

Besides for survival, clean water is also an indispensable factor for socio-economic development. However, the lack of safe drinking water supplies is a worldwide issue in the past decades [1-2]. In the developing regions many people still consume water directly from sources such as rivers, lakes etc. which are vulnerable to pathogenic microorganisms that will lead to diseases such as cholera, dysentery and diarrhea [3-4].

To date, conventional water treatment has become a common technique used to treat water in order to achieve drinking water, which meets the standard quality. However, this system cannot effectively answer the water quality standard of modern era, which is increasingly stringent. In addition, the presence of residual chlorine as a disinfectant used in conventional water treatment processes can react with natural organic matters (NOM) and produce carcinogenic disinfection by-product precursors (DBPs) such as trihalomethane, haloacetic acids, and so on. These DBP compounds are also capable of causing miscarriage and nervous system complications $[3,5-6]$

To overcome this issue, a considerable huge number of studies suggest that membrane separation process can be employed as an alternative method for drinking water treatment [7-10] because of its many advantages, such as compactness, capable in exterminating pathogens, turbidities, organic materials etc., as well as low energy consumption, eco-friendly and ease of operation.

The operational principle of filtration using membrane technology involves complex physical, chemical and biological interactions between the surface of the membrane and the contaminants. These interactions affect each other, resulting in the occurrence of fouling. Fouling on the membrane has become a crucial problem in the use of low pressure membranes for drinking water production [9, 11-12].

Surface modification by enhancing membrane hydrophilicity is one of the strategy that can be used to reduce fouling. Since most of polymeric membranes are extremely hydrophobic, for example PVDF which contact angle is over $90^{\circ}$ [13]. This hydrophobic nature causes hydrophobic-hydrophobic interaction between membranes and common foulants [12]. Modification of membrane surfaces with more hydrophilic compounds may reduce the interaction of fouling by captivating a securely-bonded water layer on the membrane surface in which it will hinder the adhesion of foulants [14-15].

Polydopamine (PDA) is one of the most hydrophilic substances and it has a super adhesion characteristic which makes it can be deposited on various types of substrate. PDA has been widely utilized in membrane separation for water and wastewater treatment to reduce fouling. However, there is a large volume of published studies reporting that modification with PDA, on the

\footnotetext{
*orresponding author: sri.mulyati@unsyiah.ac.id
} 
other hand, decreased the permeation performance of the membrane [16-17]. This decline in permeation performance is thought to be caused by the width of the PDA coating on the membrane surface, which immediately leads to pore blockage. For porous membranes, pore size reduction can be a major factor in decreasing pure water permeation. In addition, changes in pore size of the membrane also affect the fouling behavior [17-18].

Therefore, this study aims to find out the best modification conditions such as coating duration of PVDF membrane with PDA so that it can be known which conditions produce membranes with the best performance, with high invulnerability to fouling and stable permeation performance. In this work, the base PVDF membrane was firstly prepared by phase inversion method using DMAc as solvent. The prepared membrane was then immersed in a $0.5 \mathrm{mg} / \mathrm{ml}$ polydopamine solution with various duration of 2, 6 and 24 hours. The morphology, composition, mechanical strength of the membranes were characterized. The performance tests were also conducted such as pure water permeability, and selectivity on humic acid (HA) as a mimic of natural organic matter.

\section{Materials and methods}

\subsection{Materials}

Polyvinylidene fluoride (PVDF) was used as a base polymer. N.N-dimethylacetamide (DMAc) as the solvent, Poly Ethylene Glycol (PEG) of 20kDa was used as pore forming, humic acid (HA) was used as a Natural Organic Matter (NOM) model sample, and distilled water was utilized as a non-solvent. In addition, (3aminopropyltriethoxysilane), dopamine, Isopropyl Alcohol, Tris-HCl were also involved in the work as additives for modification. Furthermore, casting knife (2mm) and glass plate were used to fabricate the membrane. A dead-end module was used in the filtration experiment.

\subsection{Preparation of PVDF membrane}

The pristine PVDF membranes was fabricated by the non-solvent induced phase separation (NIPS) technique. The main polymer and additive PEG were mixed altogether with DMAc with ratio of $20 \%$ polymer: $5 \%$ PEG: $75 \%$ DMAc. The solution afterward agitated vigorously at temperature of $50^{\circ} \mathrm{C}$ until a homogenous solution is obtained. This dope solution was then casted on a glass plate with thickness of $2 \mathrm{~mm}$ and immersed into a coagulating bath containing non-solvent at room temperature condition. The fabricated membranes were stored in distilled water for 24 hours at room temperature to remove the remaining solvent from the membrane.

\subsection{Modification of PVDF membrane}

\subsubsection{Preparation of polydopamine}

Dopamine hydrochloride in the desired amount was dissolved in a $15 \mathrm{mM}$ Tris-HCL buffer ( $\mathrm{pH} 8.8$ ). After mixing, the initially transparent solution was then discolored to brown due to reaction of dopamine with oxygen. This brownish color indicates that polydopamine (PDA) has been formed [19].

\subsubsection{Preparation of PDA/PVDF membrane}

The modification of the membrane was conducted by adapting the procedure used by Feng et al [20]. The virgin PVDF holding membrane was first immersed into polydopamine solution for 2, 6 and 24 hours. Following that, the membranes were washed with distilled water and soaked into Isopropyl Alcohol for 30 minutes. In following sections the membranes will be codenamed as PVDF (nonmodified), PDA-2, PDA-6 and PDA-24 for immersion time 2, 6 and 24 hours, respectively.

\subsection{Membrane characterization and performance test}

\subsubsection{Morphology test}

Morphology of the membranes was analyzed by Scanning Electron Microscopy (SEM). The procedure was carried out by trimming the membrane piece into a smaller size and then frozen.

\subsubsection{Membrane composition analysis}

The presence of functional groups in the membrane was evaluated by Fourier Transform Infra-Red Spectroscopy (FTIR) (Shimadzu, model IRP Prestige21, serial no. A21004802668LP). The membrane specimens were allowed at room temperature to evaporate the content of water. The specimens were then placed on a sample holder. The recorded data was in $\%$ transmittance at spectrum range of 500 to $4000 \mathrm{~cm}^{-1}$.

\subsubsection{Water flux test}

The sample solution was fed into the pressure-driven membrane module, the flux generated at each experiment was collected and recorded every 5 minutes for 30 minutes. The experiment was performed three times on each type of membrane. The flux of pure water was calculated by means of following equation (1).

$$
J=\frac{\Delta V}{\Delta t} x A
$$

$\mathrm{J}$ is the generated flux $\left(\mathrm{Lm}^{-2}\right.$ hour $\left.{ }^{-1}\right) ; \Delta \mathrm{V}$ is the volume of permeate $(\mathrm{L}), \Delta \mathrm{t}$ is the time of filtration (hour) and $\mathrm{A}$ is the membrane surface area $\left(\mathrm{m}^{2}\right)$. 


\subsubsection{Solute rejection}

Humic acid as a representative of common natural organic matters (NOMs) in water was used to assess the rejection properties of the PDA-coated and pristine PVDF membranes. The HA-containing feed solution was initially prepared in a stock solution of $1000 \mathrm{ppm}$ by dissolving 1 gram of humic acid in 1 litter of deionized water [21]. This solution was then diluted to $10 \mathrm{ppm}$ for the experiment. The procedure performed was exactly like that conducted in the pure flux test experiment. The concentration of collected permeate was then measured by using UV-Vis Spectrophotometry. Next, the obtained concentration was used to calculate the rejection value using equation (2):

$$
R \%=\left(1-\frac{C_{P}}{C_{f}}\right) x 100
$$

Where, $\mathrm{R}$ is solute rejection (\%), $\mathrm{C}_{\mathrm{p}}$ and $\mathrm{C}_{\mathrm{f}}$ are concentration of permeate and feed $(\mathrm{mg} / \mathrm{ml})$, respectively.

\section{Results and discussion}

\subsection{Membrane morphology}

The effect of PDA coating on PVDF membrane was analyzed using Scanning Electron Microscopy (SEM). The observations were made on the surface and crosssection structures of the membranes before and after modification. Figure 1 shows the surface imaging result. It is seen that the length of immersion time affects the thickness of the coating. On uncoated PVDF membrane, pore distribution is still apparent. As the time of immersion in polydopamine solution increases, the membrane surface becomes denser, resulting from the increasing number of PDA particles deposited on the membrane surface [22-24].
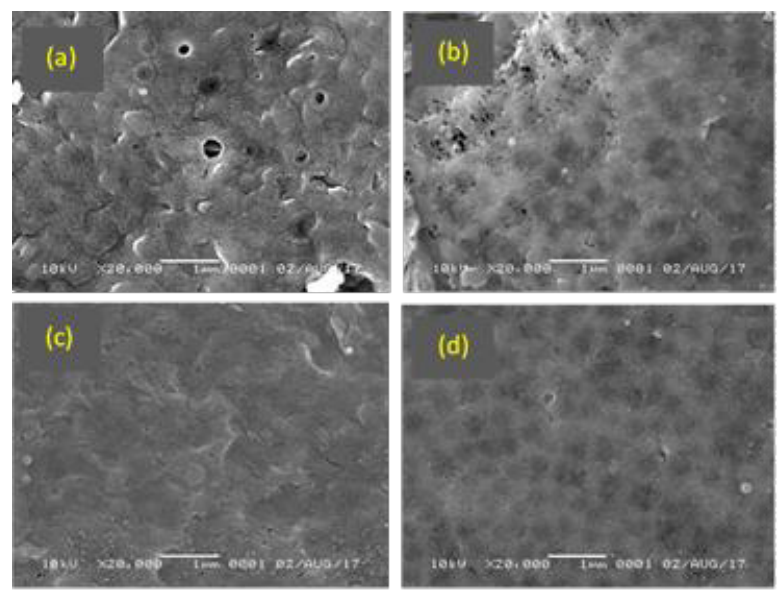

Fig. 1. SEM images of PVDF membrane (a) without PDA coating, (b) 2 hours, (c) 6 hours, and (d) 24 hours of coating with PDA.

Figure 2 presents the cross-section view of the membrane without and after coating for 24 hours. First of all, the image confirms that the prepared membrane has an asymmetrical structure in which it consists of two layers, upper layer with a denser pore composition and the bottom layer with a finger-like pore structure [25]. There is no change in the inner pore and the membrane matrix both before and after the coating. This is because the modification was performed by surface-coating therefore only the surface of the membrane is directly involved with polydopamine. The same finding is also reported by Saraswathi and co-researchers in their recent study [26].
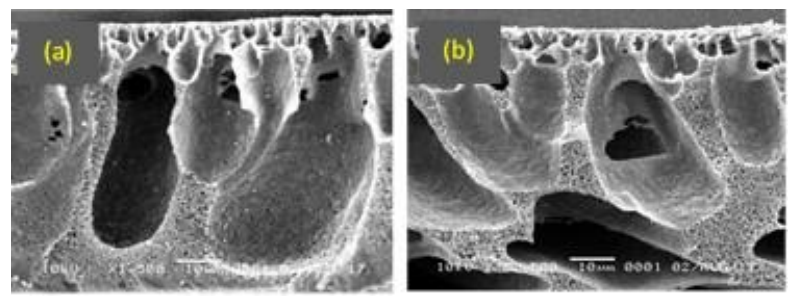

Fig. 2. Cross-section SEM images of (a) pure PVDF membrane and (b) coated with PDA for 24 hours.

It can be seen from the Figure 2 that the pores on the surface of coated membrane are more covered in comparison to those without modification, this is because PDA coating can cause pore-clogging. The pore blockage of the membrane is a negative effect of the coating modification method, as it will have an impact on the decline in permeation performance during filtration process.

\subsection{Membrane composition analysis}

FTIR analysis result as shown in Figure 3 confirms that polydopamine has been successfully introduced on the surface of PVDF membrane. The most particular characteristic of amine compound is the emergence of peak at spectrum range of $3300-3600 \mathrm{~cm}^{-1}$. This peak appears due to effect of $\mathrm{N}-\mathrm{H}$ bond stretching that usually visible in weak to medium or sometimes broad but not as broad as the O-H band from alcohol [27].

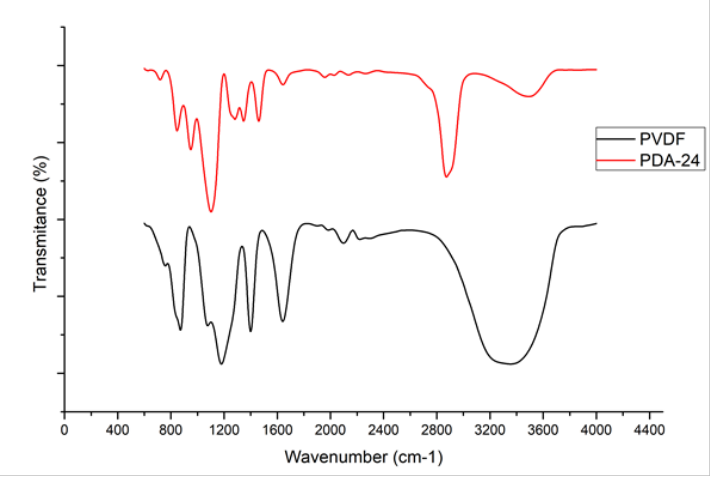

Fig. 3. FTIR Spectra of pure PVDF membrane and that of after 24 hours immersion in PDA solution.

In the same spectrum range also appears stretching of the $\mathrm{O}-\mathrm{H}$ group from the catechol group contained in polydopamine. In addition, in wavenumber $2878 \mathrm{~cm}^{-1}$ 
appears a peak from stretching of aliphatic $\mathrm{C}-\mathrm{H}$ group, and a new peak at $1509 \mathrm{~cm}^{-1}$ evidencing the presence of $\mathrm{N}-\mathrm{H}$ bending. These results are consistent with those reported in other studies [26-29].

It is worth nothing that Figure 3 only provides the spectra results of a pure PVDF membrane and that after immersion with PDA for 24 hours. The reason is because the result in membrane PDA-24 is more obvious that those of PDA-2 and PDA-6, hence it is easier for comparison. For membrane with modification for 2 and 6 hours, no apparent change were observed, allegedly because of the short duration of immersion. Moreover, since the concentration of PDA used for modification is considered not too high thus the resulting effect is not greatly noticeable.

\subsection{Water permeation performance}

Figure 4 displays the water flux of PVDF membrane without and with PDA coating. It is observed that the membrane performance in terms of permeation is improving with increasing length of immersion time. At a pressure of 0.5 bar, a pure PVDF membrane generated a flux of $2.86 \mathrm{~L} / \mathrm{m}^{2}$.hour, whereas in the modified membranes the generated fluxes were $7.59,13.19$ $\mathrm{L} / \mathrm{m}^{2}$.hour for 2, 6 hours of immersion time, respectively and slightly decreased at 24 hours immersion time which amounted to $11.44 \mathrm{~L} / \mathrm{m}^{2}$.hour. At the highest experimental pressure the flux produced by the 6-hour and 24-hour coated membranes were exceeding 50 $\mathrm{L} / \mathrm{m}^{2}$.hour, whereas the flux for pure PVDF membrane only $24.96 \mathrm{~L} / \mathrm{m}^{2}$.hour.

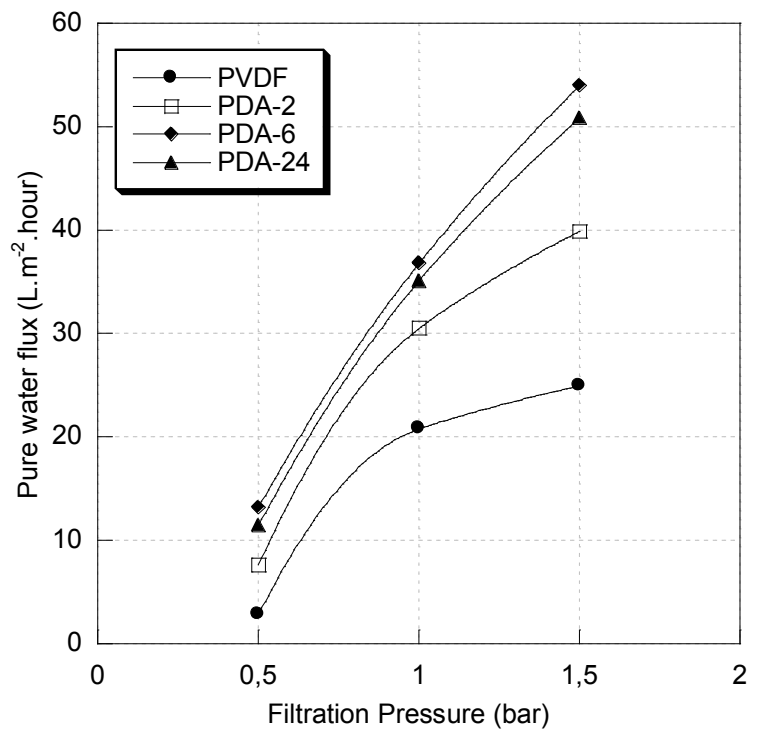

Fig. 4. Effect of PDA immersion time on membranes pure water flux (J).

The slim decline of flux in PDA-24 membrane is in accordance with earlier prediction of pore clogging by PDA coating. However, all the coated membranes generally showcased an excellent pure water flux performance in comparison to pristine PVDF allegedly because the PDA is a highly hydrophilic material due to the presence of so many compounds of catechol and amines it contains [30] as confirmed by FTIR analysis in Section 3.2. PDA coating will enhance the hydrophilicity of PVDF membrane. Hydrophilicity surface allows better interaction between material and water [26]. Unfortunately, in this research, the contact angle of fabricated membranes was not measured, so this reasoning remains a conjecture.

Table 1. The water permeability coefficients of the fabricated membranes.

\begin{tabular}{|c|c|c|}
\hline Membrane & $\begin{array}{c}\mathrm{Lp} \\
\left(\mathrm{L} / \mathrm{m}^{2} . \mathrm{h} \cdot \mathrm{bar}\right)\end{array}$ & Types \\
\hline Uncoated PVDF & 11.05 & Ultrafiltration \\
\hline PDA-2 & 16.13 & Ultrafiltration \\
\hline PDA-6 & 20.39 & Ultrafiltration \\
\hline
\end{tabular}

Table 1 provides a summary of membrane coefficient of permeability (Lp) which obtained from the slope of the flux vs operating pressure plot. According to Mulder [31], membranes with Lp between 10 to $50 \mathrm{~L} / \mathrm{m}^{2}$.h.bar belong in the ultrafiltration type. Based on the results shown in the table above, it is confirmed that all the fabricated membranes in this research are classified in the ultrafiltration group.

\subsection{Humic acid rejection}

Figure 5 presents the results of performance evaluation in terms of solute rejection for membranes before and after modification with PDA. It appears that the percentage of HA rejection increases after the membrane is modified with PDA. The rejection of humic acid generated from separation experiment using PVDF, PDA-2, PDA-6 and PDA-24 are 66.34\%, 79.92\%, $86.78 \%$ and $91.23 \%$, in respective order. This is because the PDA particles on the surface of the membrane cover the pores hence the absorption area is getting smaller. This hinders the HA molecules passing through the membrane [32].

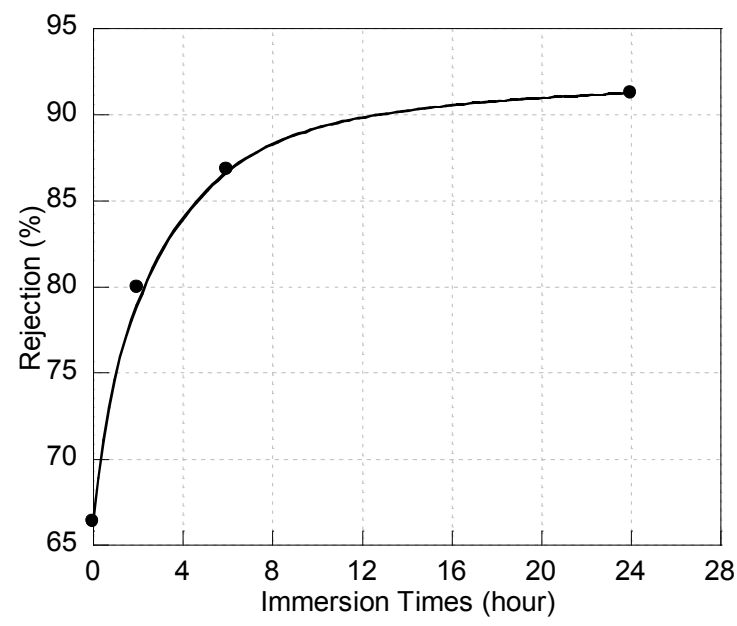

Fig. 5. Effect of PDA immersion time on humic acid rejection. 
In addition, the hydrophilic functional groups of polydopamine causing the membrane to become hydrophilic. Increased hydrophilicity of the membrane surface inhibits interaction with hydrophobic HA molecules [26].

\subsection{Mechanical properties}

Mechanical properties are prominent parameters to consider in judging how far the membrane is able to handle the workload. Workload is the amount of distortion that the membrane can sustain without experiencing damaged. During operation, any kind of membrane such as for ultrafiltration process in water or wastewater treatment will have to endure the stress and strain be it from feed containing contaminant or from the given driving force which will cause the membrane to be destructed in certain period of time. Therefore, it is desireable for the membrane to have good mechanical traits. The mechanical properties of a membrane is generally represented by tensile strength and elongation at break.

Table 2. Mechanical properties of pristine and PDA-modified PVDF membranes.

\begin{tabular}{|c|c|c|}
\hline Membranes & $\begin{array}{c}\text { Tensile } \\
\text { Strength }\end{array}$ & Membranes \\
\hline PVDF & 7.32 & 169.60 \\
\hline PDA-2 & 13.21 & 207.48 \\
\hline PDA-6 & 12.93 & 311.78 \\
\hline PDA-24 & 6.72 & 356.90 \\
\hline
\end{tabular}

The result of tensile testing for fabricated membranes as shown in Table 2. In general, it appears that the PDA coating enhanced the mechanical strength of the PVDF membrane. The original PVDF membrane tensile was $7.32 \mathrm{kgf} / \mathrm{mm}^{2}$, after being immersed in polydopamine solution for two hours, the tensile increased to 13.21 $\mathrm{kgf} / \mathrm{mm}^{2}$, but after 6 hours of coating, the tensile decreased slightly to $12.93 \mathrm{kgf} / \mathrm{mm}^{2}$ and considerably declined again on dipping for 24 hours.

Tensile strength is the force required to break or cause complete separation of the specimen in a linear direction. The tensile detoriation of PDA-coated PVDF membrane is explainable. It is possible when a large number of hydrophilic functional groups is added to the membrane can decrease the mechanical strength of the membrane. As is the case of a study conducted by Zhu et al, tensile from cross linked membrane initially increased because the additive used caused the transition of support layer from having finger-like morphological to sponge-like, causing the membrane to have a more compact structure. However, when the additive concentration used increased, the tensile decreased sharply [15]. In addition, Hwang and research team [33] reported that overloading of PDA as additive may cause heterogeneity between PDA coating and surface of the substrate which leads to miscibility complication impacting on the interfacial bonding and load transfer.

In contrast to tensile, elongation at break of the membrane improved with the increasing immersion time in polydopamine solution. Elongation at break is defined as the percentage of distance obtained when the membrane is stretched from its original size until it is broken. In short, the greater the elongation value of the membrane means the more flexible and better the membrane characteristic is because it means the membrane is not easily broken. For PVDF membrane, even without modification, the elongation at break was up to $169.60 \%$. It is considered a big number because PVDF basically has good mechanical strength due to its degree of crystallinity ( $\mathrm{Tg}$ ) in comparison to other polymers [34].

After immersion with PDA for 24 hours, the elongation at break of the PVDF membrane reached $356.90 \%$. This result is in accordance with previous research conducted by Jiang and co-workers [35], in which the PVDF membrane which modified with PDA by blending technique showcased a greater elongation at break than the original PVDF membrane. This increase in elongation at break is attributed by the PDA particles existing in the modified membrane which capable in absorbing energy during the tensile deformation process, resulting in the diminishing of membrane brittleness or the membrane became more ductile [35].

\section{Conclusions}

The modification of PVDF membrane by coating using polydopamine (PDA) has been studied. Modification was conducted by varying the immersion time of $0,2,6$ and 24 hours. It has been proven that the duration of the immersion time has an effect on the characteristics and performance of the membrane. The longer-immersed membranes in the dopamine solution have a thicker layer and denser pore on the surface. Overall, the coated membranes produced larger flux than those without modification. This is because PDA coating make the membrane surface to be more hydrophilic. Although without contact angle analysis, membrane hydrophilicity after modification can be observed from the emergence of hydrophilic groups such as catechol and amines carried by PDA as evidenced by the FTIR analysis results. However, for the 24-hour immersion membrane, the pure water flux declined due to pore clogging on the surface of the membrane by PDA particles. In the case of humic acid rejection performance, the longer the coating time the greater the percent of rejection obtained. In addition, coating with a PDA also increases the mechanical strength of the membrane as seen from the tensile and elongation at break values.

\section{References}

1. N.P. Sisto, A.I. Ramírez, I. Aguilar-Barajas, V. Magaña-Rueda, Phys. Chem. Earth Part A/B/C 91, 2 (2016) 
2. P. Goovaerts, Sci. Total Environ. 58, 66 (2017)

3. J. Han, X. Zhang, J. Liu, X. Zhu, T. Gong, J. Environ. Sci. 58, 83 (2017)

4. S. Mulyati, N. Arahman, Syawaliah, Mukramah, IOP Conference Series: Materials Science and Engineering, 180, 012128 (2017)

5. M.J. Plewa, S.D. Richardson, J. Environ. Sci. 58, 1 (2017)

6. R.A.A. Carter, C.A. Joll, J. Environ. Sci. 58, 19 (2017)

7. L. Bai, H. Liang, J. Crittenden, F. Qu, A. Ding, J. Ma, X. Du, S. Guo, G. Li, J. Membr. Sci. 492, 400 (2015)

8. X. Cheng, H. Liang, A. Ding, F. Qu, S. Shao, B. Liu, H. Wang, D. Wu, G. Li, G, J. Membr. Sci. 505, 15 (2016)

9. J. Winter, W. Uhl, P.R. Bérubé, Water Res. 104, 418 (2016)

10. S. Mulyati, N. Arahman Mukramah, Syawaliah, S Mulyati, N Arahman, IOP Conference Series: Materials Science and Engineering, 180, 012130 (2017)

11. A. Charfi, H. Jang, J. Kim, Bioresour. Technol. 240, 106 (2017)

12. J. Lee, S. Jeong, Y. Ye, V. Chen, S. Vigneswaran, T. Leiknes, Z. Liu, Z, Sep. Purif. Technol. 176, 323 (2017)

13. J. Wang, L. Zheng, Z. Wu, Y. Zhang, X. Zhang, J. Membr. Sci. 497, 183 (2016)

14. C. Cheng, S. Li, W. Zhao, Q. Wei, S. Nie, S. Sun, C. Zhao, J. Membr. Sci. 417, 228 (2012)

15. K. Zhu, S. Zhang, J. Luan, M. Mu, Y., Du, G. Wang, J. Membr. Sci. 539, 116 (2017)

16. B.D. McCloskey, H.B. Park, H. Ju, B.W. Rowe, D.J. Miller, B.J. Chun, K. Kin, B.D. Freeman, Polymer, 51, 3472 (2010)

17. W. Zhang, Y. Ying, J. Ma, X. Guo, H. Huang, D. Liu, C. Zhong, C., J. Membr. Sci. 527, 8 (2017)

18. K.Y. Kim, E. Yang, M.Y. Lee, K.J. ChaeC.M. Kim, I.S. Kim, Water Res. 54, 62 (2014)

19. M. Vaselbehagh, K. Karkhanechi, S. Mulyati, R. Takagi, H. Matsuyama, H., Desalination, 332, 126 (2014)

20. K. Feng, L. Hou, B. Tang, P. Wu, J. Membr. Sci. Science, 490, 120 (2015)

21. A. Mehrparvar, A. Rahimpour, M. Jahanshahi, J. Taiwan Inst. Chem. Eng. 45, 275 (2014)

22. V. Ball, D. Del Frari, V. Toniazzo, D. Ruch, J. Colloid Interface Sci. 386, 366 (2012)

23. J. Ou, J. Wang, S. Liu, J. Zhou, S. Ren, S.Yang, 2009. Appl. Surf. Sci. 256, 894 (2009)

24. F. Li, J. Meng, J. Ye, B. Yang, Q. Tian, C. Deng, Desalination, 344, 422 (2014)

25. N. Arahman, T. Maimun, Mukramah, Syawaliah, AIP Conference Proceedings, 1788, 030018 (2017)
26. M.S.A. Saraswathi, R. Kausalya, N.J. Kaleekkal, D. Rana, A. Nagendran, A., J. Environ. Chem. Eng. 5, 2937 (2017)

27. R.A. Zangmeister, T.A. Morris, M.J., Tarlov, Langmuir, 29, 8619 (2013)

28. B. Fei, B. Qian, Z. Yang, R. Wang, W.C., Liu, C.L. Mak, J.H. Xin, Carbon, 46, 1795- (2008)

29. H. Guo, Y. Deng, Z. Tao, Z. Yao, J.Wang, C. Lin, T. Zhang, B. Zhu, C.Y. Tang, Environ. Sci. Technol. Lett. 3, 332 (2016)

30. Y. Liu, K. Ai, L. Lu, Chem. Rev. 114, 5057 (2014)

31. Mulder, J. Springer Science \& Business Media (2012)

32. N. Arahman, S. Mulyati, S., IOP Conference Series: Materials Science and Engineering, 180, 012129 (2017)

33. S.H. Hwang, D. Kang, R.S. Ruoff, H.S. Shin, Y.B. Park, Y.B., ACS nano, 8, 6739 (2014)

34. A. Behboudi, Y. Jafarzadeh, Y., R. Yegani, 2017. J. Membr. Sci., 534, 18 (2017)

35. J.H. Jiang, L.P. Zhu, H.T. Zhang, B.K. Zhu, Y.Y. Xu, J. Membr. Sci. 457, 73 (2014) 